
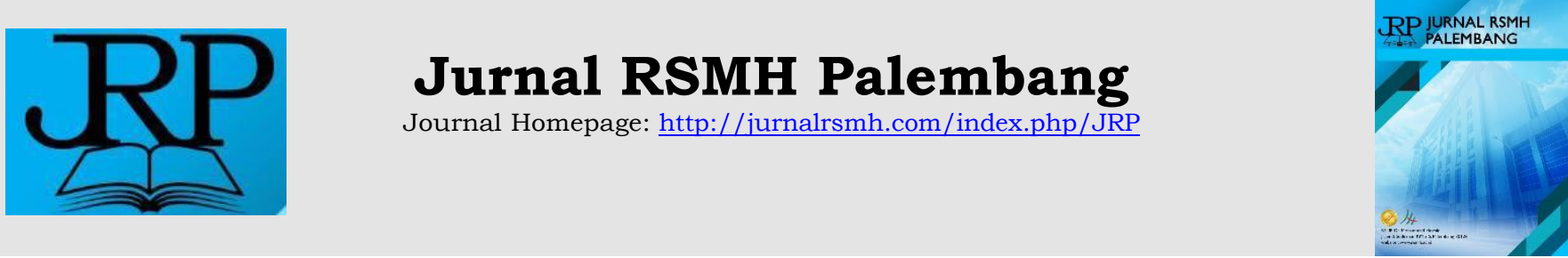

\title{
Black Dot Type Capitis Tinea Appreciates Bacterial Foliculitis
}

\section{Radema Maradong Ayu Pranata ${ }^{1}$, Rusmawardiana ${ }^{1}$, Fifa Argentina ${ }^{1}$}

${ }^{1}$ Department of Dermatology and Venereology, Faculty of Medicine, Sriwijaya University, Palembang

\author{
A R T I C L E I N F O \\ Keywords: \\ Black dot \\ Bacterial Folliculitis \\ Tinea Capitis
}

\section{Corresponding author:}

Radema Maradong Ayu Pranata

E-mail address:

radema.pranata@gmail.com

All authors have reviewed and approved the final version of the manuscript.

https://doi.org/10.37275/JRP.v1i1.3

\begin{abstract}
A B S T R A C T
Tinea capitis is a superficial fungal infection of the scalp and hair, which is seen predominantly in children. In adults, it is usually related to immunocompromised patients and have an atypical features. In patients with end stage renal disease (ESRD), uremia is associated with immune suppression due to the impact of uremic milieu. All specimens of tinea capitis should be examined for microscopy, wood's lamp and culture. Reported a case of 50-year-old male, animal husbandry, presented with itchy papules, pustules, patch alopecia and a hair loss for 6 months. Dermatologic features showed papules, pustules, patch alopecia and black dot. The patient treated with ketoconazole shampoo for 3 weeks without any improvement. He had an ESRD for 2 years. Gram stain examination and culture showed no bacteri. Wood's lamp examination showed no fluorescent. Potassium hydroxide $(\mathrm{KOH}) 10 \%$ from scalp scrapings and $\mathrm{KOH} 20 \%$ from hair showed a fungal elements, which support diagnosis of black dot tinea capitis. The patient treated with griseofulvin tablet $500 \mathrm{mg}$ twice a day for 8 weeks, cetirizine tablet $10 \mathrm{mg}$ once daily and $3 \mathrm{x} /$ week of ketoconazole shampoo $2 \%$ showed improvement in clinical features and microscopic evaluation.
\end{abstract}

\section{Introduction}

Tinea capitis is a superficial fungal infection of the scalp and hair, especially in children aged 3-14 years. ${ }^{1,2}$ The fungistatic effect of fatty acids in sebum may explain the decreased incidence of tinea capitis after puberty. ${ }^{2,3}$ Tinea capitis in adults most occur in immunocompromised patients. ${ }^{1}$ For example, in patients with end-stage chronic kidney disease (CKD) that is associated with decreased immunity due to uremia. 4

The classification of superficial fungi according to habitat consists of anthrophopilic, zoophilic and geophilic. The classification provides information about the source of infection. Anthrophopilic disease is transmitted by direct contact of infected skin or hair. For example cloth, comb, socks and towels. Zoophilic transmitted from animals, such as cats, dogs, horses, birds or cows. Geophilics cause sporadic infections due to direct contact with the soil. Tinea capitis is often caused by Tricophyton and Microsporum species with clinical features of inflammatory, non-inflammatory, favus, and black dot types.2

Inflammatory type tinea capitis is usually caused by zoophilic or geophilic ectothrix pathogens, such as $M$. canis, M. gypseum and T. verrucosum. Pathogens $M$. gypseum and T. verrucosum did not fluoresce on Wood's lamp examination, but $M$. canis would fluoresce green yellow. The non-inflammatory type is often caused by ectothrix anthrophopilic pathogens such as $M$. audouinii or $M$. ferrugineum. The clinical presentation of the non-inflammatory type is a gray patch with yellowish green fluorescence. $T$. schoenleinii is the most common type of favus, although it can also be caused by $T$. violaceum and $M$. gypseum. The clinical features of the favus type are perifollicular erythema patches with yellow crust (scutula) and smell like cheese or mousy odor. Tinea favus will fluoresce blue-gray if caused by $T$. schoenleinii. Black dot type tinea capitis is 
usually caused by the antrophophilic endothrix pathogens T. tonsurans and T. violaceum. Broken hair on the scalp causes alopecia patches with a black dot appearance. Black dot type tinea capitis tends to have minimal inflammation, can form follicular pustules, nodules, furuncles or in rare cases kerion. This type of tinea capitis does not have fluorescence. ${ }^{2,5}$

All specimens from cases of tinea capitis should be subjected to microscopic examination and culture whenever possible. 6 The study by Gupta et al. (2014) reported a high sensitivity $(73.33 \%)$ on $\mathrm{KOH}$ examination, so that $\mathrm{KOH}$ can be used as the definitive procedure for screening and diagnosis of dermatophyte infections. ${ }^{7}$ The $\mathrm{KOH}$ test results can give false negative results in about $15 \%$ of cases, so patients who have a clinical picture of dermatophytosis must still be treated even though the $\mathrm{KOH}$ test results are negative. ${ }^{2,8}$

\section{CASE REPORT}

A 50 year old man working as a farm employee complained of erythematous papules, pustules, alopecia patches with black dot and hair loss since 6 months ago (Figure 1). The patient has been using ketoconazole shampoo for 3 weeks, but there is no improvement. The patient had a 2 -year history of CKD and had undergone hemodialysis. No other family members have had similar lesions. Wood's lamp examination showed no fluorescence. $\mathrm{KOH}$ examination of $10 \%$ of scalp scrapings revealed hyphae and arthrospores (Figure 2a). KOH examination of $20 \%$ of the hair showed endothrix spores with magnification of 10x (Figure 2b) and 40x (Figure 2c). Culture with Saboroud dextrose agar was negative.

Patients were treated with $2 \times 500 \mathrm{mg}$ of griseofulvin tablets, $2 \%$ ketoconazole shampoo used 3 times / week and $1 \times 10 \mathrm{mg}$ cetirizin tablets. Treatment was carried out for 8 weeks and clinical improvement was obtained (Figure 3). Both clinical and microscopic follow-up observations with $\mathrm{KOH}$ examination showed improvement
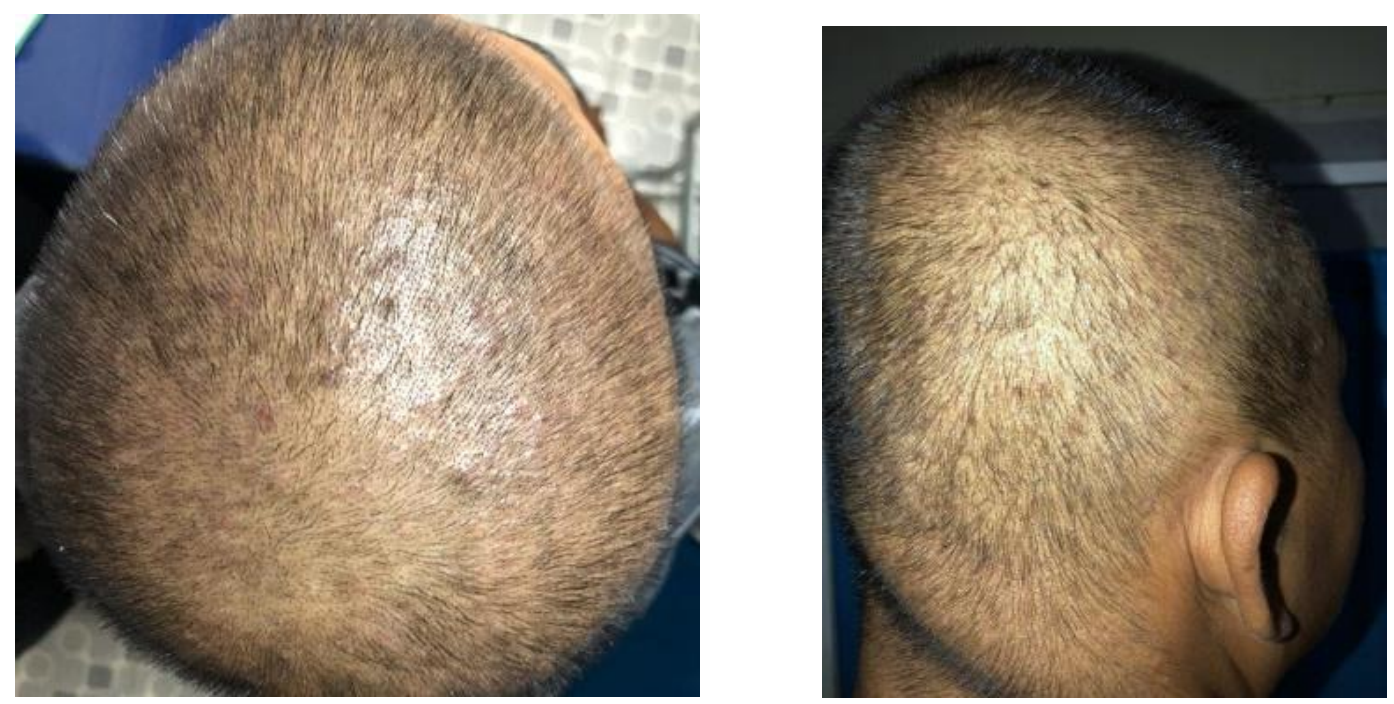

Figure 1. Baseline clinical manifestations of papules, pustules, black dots, patches of alopecia 

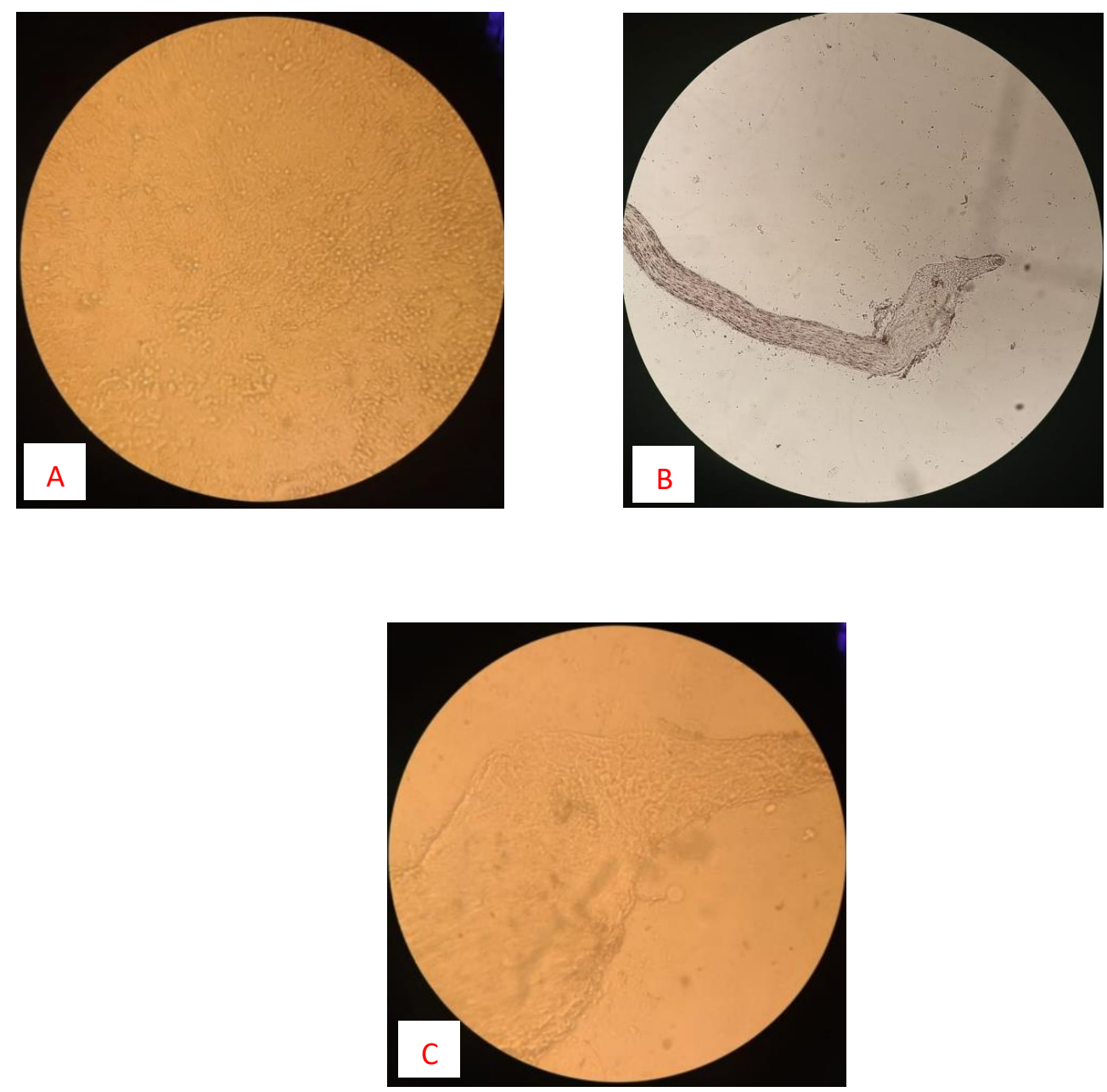

Figure 2. (a) Scalp scrapings (b) endothrix spores at 10x magnification (c) endothrix spores at 40x magnification
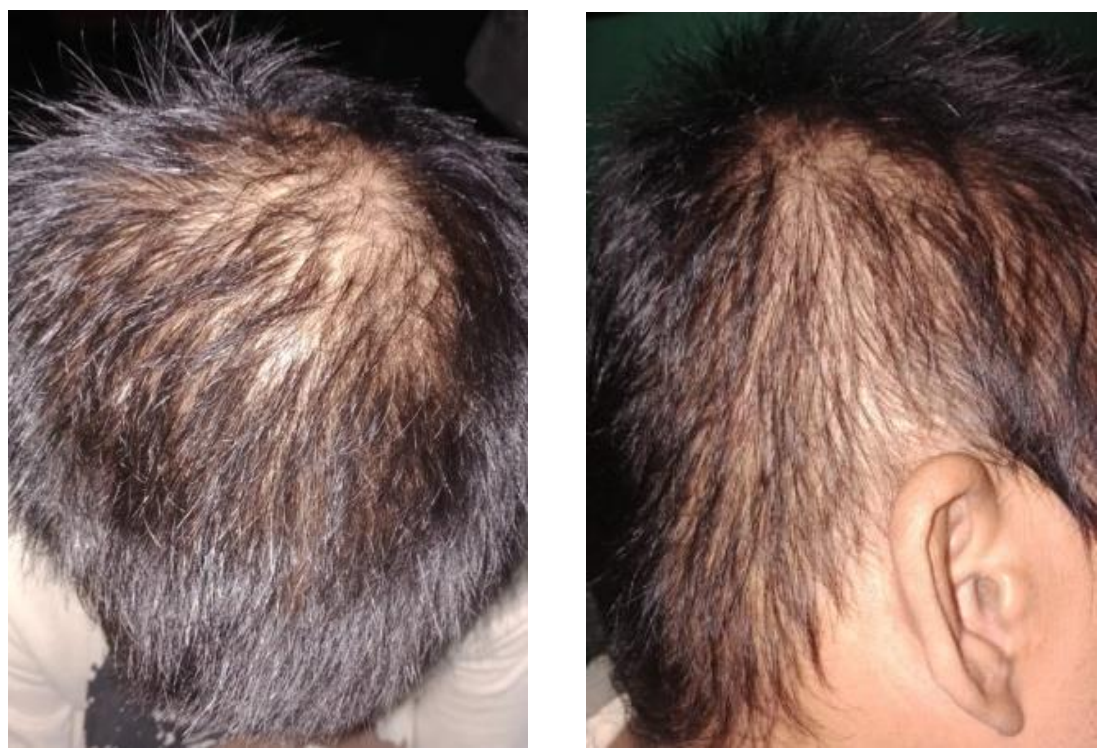

Figure 3. Clinical manifestations after 8 weeks of therapy

\section{Discussion}

In this case, a man with black dot type tinea capitis that resembled folliculitis was reported. Tinea capitis is a rare dermatophytosis in adults (range $3-11 \%$ of all 
cases). Most cases occur in immunocompromised patients. 9 The comorbid factor in these patients is endstage CKD, which affects the immune system. Research by Narain et al (2016) on patients with end-stage CKD there are a range of $2.2 \%$ of 150 patients suffering from tinea capitis. In CKD, uremia occurs due to uremic milieu, resulting in a decrease in the immune system. ${ }^{4}$

The features of the lesions in this patient were erythematous papules, alopecia patches with black dot and pustules that resembled bacterial folliculitis. The results of Gram stain did not show the presence of Gram positive or negative bacteria. Wood's lamp examination showed no fluorescence. $\mathrm{KOH}$ examination of $20 \%$ of hair specimens revealed fungal elements in the hair shaft. $\mathrm{KOH}$ examination of $10 \%$ of scalp scrapings revealed fungal hyphae and arthrospores. The fungal culture in this patient was negative.

In adults, tinea capitis caused by ectothrix or endothrix fungal pathogens can produce atypical features such as alopecia and dermatitis that mimic bacterial folliculitis. Therefore, further tests are needed such as examining Wood's lamp, $\mathrm{KOH}$ and culture. Examination of Wood's lamp (365 nm) can show pteridine fluorescence in hair infected with ectothrix fungal pathogens. The fluorescent hair was then removed for $\mathrm{KOH}$ examination and culture. The results of the $20 \% \mathrm{KOH}$ examination had a specificity value of $91 \%$ and a sensitivity of $91.9 \%$. Therefore, doctors can start treatment with just $20 \% \mathrm{KOH}$ examination results without having to do culture.10,11 In some superficial mycoses, the microscopic image of fungal elements is very distinctive so that culture is not required to build a diagnosis. ${ }^{5}$ Research by Kadhim et al (2018) reported $10.5 \%$ of 200 dermatophytosis patients having negative fungal culture results. This occurs because of differences in staff skills and the quality of sampling. 10 Another examination that can be done is a biopsy for histopathological examination. Skin biopsy is not routinely performed in dermatophytosis. A biopsy was performed to confirm the diagnosis when administering systemic drugs in cases of recalcitrant or widespread infection. ${ }^{2}$ In this patient, the results of Wood's lamp examination, $\mathrm{KOH} 20 \%$ and $10 \%$ were sufficient to support the diagnosis of black dot type tinea capitis.

In this case report, the patient was treated with $2 \times 500 \mathrm{mg}$ griseofulvin tablets, 2\% ketoconazole shampoo 3 days / week, and 1x10 mg cetirizine tablet. Treatment was carried out for 8 weeks and showed both clinical and microscopic improvement.

One type of drug that can be used to treat tinea capitis is griseofulvin. Griseofulvin is a fungistatic drug that inhibits nucleic acid synthesis, blocks cell division at metaphase and impairs cell wall synthesis. Griseofulvin is known as the gold standard therapy for tinea capitis. ${ }^{12}$ A randomized controlled trial (RCT) by Gupta et al. (2013) reported that 8 weeks of griseofulvin treatment showed significant improvement over terbinafine. ${ }^{13}$ Evidence of resistance to griseofulvin in vitro is not available. A higher dose of griseofulvin and a longer period (12-18 weeks) may be required in infections caused by Trichophyton. Griseofulvin contraindications include severe liver disease, lupus erythematosus and porphyria.

\section{Conclusion}

Tinea capitis is more common in children. In adults, it is often associated with immunocompromised conditions, one of which is CKD and presents a picture of atypical lesions. The lesion in this patient resembled bacterial folliculitis, but $\mathrm{KOH}$ examination of $20 \%$ of the hair specimen revealed hyphae and endothrix arthrospores. This result is very important because it has a specificity value of $91 \%$ and a sensitivity of $91.9 \%$, so that therapy can be given without doing culture. The therapy in this case was griseofulvin tablets because it was considered safe for CKD patients and was the gold standard therapy. Other therapies include ketoconazole shampoo and cetirizine tablets. Both clinical and microscopic improvements occurred within 8 weeks after treatment.

\section{References}

1. Ahmad SM, Wani GM, Khursheed B. Kerion mimicking bacterial infection in an elderly patient. Indian Dermatol Online J. 2014;5(4):494-6. 
2. Schieke SM, Garg A. Superficial fungal infection. In: Goldsmith LA, Katz SI, Gilchrest BA, Paller AS, Leffell DJ, Wolff K editors. Fitzpatrick's Dermatology in General Medicine.8th ed.New York: McGraw Hill.2012.p.2277-88.

3. Sacchidanand S, Savitha AS, Aparna AD, Shilpa K. Significance of scraping scalp lesions in adults. Int $\mathrm{J}$ of Trichology.2012;4(1):48-50

4. Narain U, Gupta A. Superficial fungal infections in end stage renal disease patients. Int $J$ of Advances in Medicine.2016;3(4):10437.

5. Hay RJ, Ashbee HR. Fungal infections. In: Griffiths CEM, Barker J, Bleiker T, Chalmers $\mathrm{R}$, Creamer D, editors. Rook's Textbook of Dermatology. 9th ed. Oxford: John Wiley and Sons; 2016.p.32.1-.41.

6. Fuller L.C, Barton R.C, Mustapa M, Proudfoot L.E, Punjabi S.P, Higgins E.M. British association of dermatologists' guidelines for the management of tinea capitis 2014. $\mathrm{Br} \mathrm{J}$ Dermatol. 2014; 171: 454-63.

7. Sarika G, Purva A, Rahul R, Saksham G. Prevalence of dermatophytic infection and determining sensitivity of diagnostic procedures. Int J Pharm Pharm.2014;6(3):358.

8. Sahoo AK, Mahajan R. Management of tinea corporis, tinea cruris and tinea pedis: a comprehensive review. Indian Dermatol Online J.2016;7(2):77-86.

9. Tangjaturonrusamee C, Piraccini BM, Vincenzi C, Starace M. Tinea capitis mimicking folliculitis decalvans. Mycoses.2010;54:87-88.

10. Kadhim OH. The incidence of dermatophytosis in Babylon Province, Iraq. Med $\mathrm{J}$ Babylon.2018;15(3):234-7.

11. Ardakani ME, Ghaderi N, Kafaii P. The diagnostic accuracy of potassium hydroxide test in dermatophytosis. JBCM.2016;5(2):4-6.

12. Adisty DR, Astari L. Tinea capitis favus like appearance: problem diagnosis. Berkala Ilmu
Kesehatan Kulit dan Kelamin.2017;29(3):26470 .

13. Gupta AK, Drummond-Main C. Meta-analysis of randomized, controlled trials comparing particular doses of griseofulvin and terbinafine for the treatment of tinea capitis. Pediatr Dermatol.2013;30:1-6. 